\title{
Hedgehog theory via Euler calculus
}

\author{
Yves Martinez-Maure
}

\begin{abstract}
Hedgehogs are (possibly singular and self-intersecting) hypersurfaces that describe Minkowski differences of convex bodies in $\mathbb{R}^{n+1}$. They are the natural geometrical objects when one seeks to extend parts of the Brunn-Minkowski theory to a vector space which contains convex bodies. There is a close relationship between Minkowski addition and convolution with respect to the Euler characteristic. In this paper, we extend it to hedgehogs with an analytic support function. In this context, resorting only to the support functions and the Euler characteristic, we give various expressions for the index of a point with respect to a hedgehog. Finally, we present some applications.
\end{abstract}

Keywords and phrases: Hedgehogs, convex bodies, Brunn-Minkowski theory, Euler characteristic, Euler integration, index, mixed volumes

\section{Contents:}

0 . Introduction

1. Hedgehog theory

2. Euler calculus

3. Main results

4. Proof of the results

5. Further remarks and applications

\section{Introduction}

Classical hedgehogs are (possibly singular and self-intersecting) hypersurfaces that describe differences of convex bodies with $C^{2}$ support functions in $(n+1)$-Euclidean vector space $\mathbb{R}^{n+1}[12]$. Many notions from the theory of convex bodies carry over to hedgehogs and quite a number of classical results find their counterparts. Of course, a few adaptations are necessary. In particular, areas and volumes have to be replaced by their algebraic versions, which can take negative values. The (algebraic) $(n+1)$-dimensional volume of a hedge$\operatorname{hog} \mathcal{H} \subset \mathbb{R}^{n+1}$ is defined as the integral over $\mathbb{R}^{n+1} \backslash \mathcal{H}$ of the Kronecker index, say $i_{\mathcal{H}}(x)$, of $x \in \mathbb{R}^{n+1} \backslash \mathcal{H}$ with respect to $\mathcal{H}: i_{\mathcal{H}}(x)$ can be regarded as the algebraic intersection number of almost every oriented half-line with origin $x$

2010 MSC: 28E99, 52A20, 52A30, 52A39, 53C65 
with the hypersurface $\mathcal{H}$ equipped with its transverse orientation. This index is, in some sense, the corner stone of hedgehog theory. In particular, it played a key role in obtaining a counter-example to an old uniqueness conjecture of A.D. Alexandrov $[1,9]$. On the other hand, there is a well-known relationship between Minkowski addition of convex bodies and convolution with respect to the Euler characteristic $[4,23,25]$. In the framework of hedgehogs with analytic support functions, we extend this relationship to hedgehogs and interpret the Kronecker index in terms of the Euler characteristic. We then give new expressions for the Kronecker index resorting only to the support functions and the Euler characteristic.
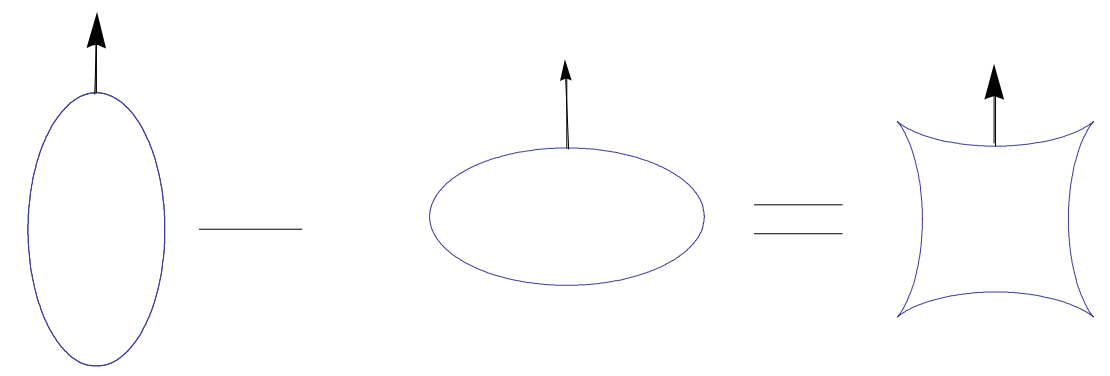

Figure 1. Minkowski difference of two convex bodies of class $C_{+}^{2}$

In $[12,14]$, the author extended the theory by regarding hedgehogs as Minkowski differences of arbitrary convex bodies (the trick is to define hedgehogs inductively as collections of lower-dimensional 'support hedgehogs'). In the polytopal case, hedgehogs are also known under the name 'virtual polytopes'. The notion of a virtual polytope was independently introduced by several authors (see, e.g., [18] or [20]).
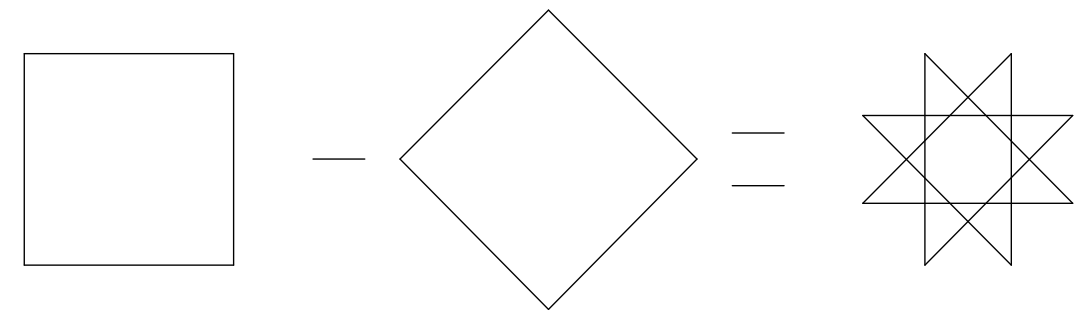

Figure 2. Minkowski difference of two squares 
The relevance of hedgehog theory can be illustrated by the following two principles [16]: 1. The study of convex bodies or hypersurfaces by splitting them judiciously (that is, according to the problem under consideration) into a sum of hedgehogs in order to reveal their structure (the study that led to the first counterexample to A.D. Alexandrov's uniqueness conjecture relied on this first principle); 2. The geometrization of analytical problems by considering real functions on the unit sphere $\mathbb{S}^{n}$ of $\mathbb{R}^{n+1}$ as support functions of hedgehogs or of more general hypersurfaces (called 'multi-hedgehogs' [11, 15]).

Hedgehog (and multi-hedgehog) theory has, of course, many applications to the Brunn-Minkowski theory. But, it also has applications to a wide variety of topics including Sturm theory [11, 15], Monge-Ampère equations [17], minimal surfaces $[13,21]$, singularity theory [5], the group of sheaves on an algebraic variety (the Picard group) [20] and planar pseudo-triangulations [19].

In this paper, we have chosen the framework of hedgehogs with analytic support functions (we shall refer to them as 'analytic hedgehogs' or ' $C{ }^{\omega}$-hedgehogs') even if some of our results still hold with a few adaptations under weaker assumptions.

Our first main result asserts that the Kronecker index of any $C^{\omega}$-hedgehog $\mathcal{H}$, which represents a formal difference $K-L$ of two convex bodies $K, L \subset \mathbb{R}^{n+1}$ of class $C_{+}^{\omega}$ (i.e., $C^{\omega}$ and with positive Gaussian curvature), is such that

$$
i_{\mathcal{H}}(x)=(-1)^{n+1}\left(\mathbf{1}_{K} * \mathbf{1}_{-L}^{o}\right)(x) \text { for all } x \in \mathbb{R}^{n+1} \backslash \mathcal{H},
$$

where $\mathbf{1}_{A}$ denotes the characteristic function over a subset $A \subset \mathbb{R}^{n+1}$, * the convolution product with respect to Euler characteristic $\chi$ and $-\stackrel{o}{L}$ the reflection of $\stackrel{o}{L}$ through the origin $0_{\mathbb{R}^{n+1}}$.

Our second main result gives the following expression of the Kronecker index of any hedgehog $\mathcal{H} \subset \mathbb{R}^{n+1}$ with support function $h \in C^{\omega}\left(\mathbb{S}^{n} ; \mathbb{R}\right)$ :

$$
\forall x \in \mathbb{R}^{n+1} \backslash \mathcal{H}, \quad i_{h}(x)= \begin{cases}1-\frac{1}{2} \chi_{h}(x) & \text { if } n+1 \text { is even } \\ \frac{1}{2}\left(\chi_{h}^{+}(x)-\chi_{h}^{-}(x)\right) & \text { if } n+1 \text { is odd }\end{cases}
$$

where $\chi_{h}(x):=\chi\left[\left(h_{x}\right)^{-1}(\{0\})\right], \chi_{h}^{-}(x):=\chi\left[\left(h_{x}\right)^{-1}(]-\infty, 0[)\right]$ and $\chi_{h}^{+}(x):=$ $\chi\left[\left(h_{x}\right)^{-1}(] 0,+\infty[)\right]$.

The paper is organized as follows. Section 1 recalls basic definitions and facts on hedgehog theory. For the convenience of the reader, Section 2 briefly summarizes basic notions and results from Euler's integral calculus. Section 3 presents the main results, Section 4 the proofs and Section 5 further remarks and applications. 


\section{Hedgehog theory}

The set $\mathcal{K}^{n+1}$ of all convex bodies of $(n+1)$-Euclidean vector space $\mathbb{R}^{n+1}$ is usually equipped with Minkowski addition and multiplication by non-negative real numbers which are respectively defined by:

$$
\begin{aligned}
& \text { (i) } \forall(K, L) \in\left(\mathcal{K}^{n+1}\right)^{2}, K+L=\{u+v \mid u \in K, v \in L\} ; \\
& \text { (ii) } \forall \lambda \in \mathbb{R}_{+}, \forall K \in \mathcal{K}^{n+1}, \lambda . K=\{\lambda u \mid u \in K\} .
\end{aligned}
$$

Of course, $\left(\mathcal{K}^{n+1},+,.\right)$ does not constitute a vector space since convex bodies cannot be subtracted in $\mathcal{K}^{n+1}$. Now, in the same way as we construct the group of integers from the set of all natural numbers, we can construct the vector space $\left(\mathcal{H}^{n+1},+,.\right)$ of formal differences of convex bodies of $\mathbb{R}^{n+1}$ from $\left(\mathcal{K}^{n+1},+,.\right)$.

Moreover, we can [14]: 1. consider each formal difference of convex bodies of $\mathbb{R}^{n+1}$ as a (possibly singular and self-intersecting) hypersurface of $\mathbb{R}^{n+1}$, called a hedgehog; 2 . extend the mixed volume $V:\left(\mathcal{K}^{n+1}\right)^{n+1} \rightarrow \mathbb{R}$ to a symmetric $(n+1)$-linear form on $\mathcal{H}^{n+1}$.

Thus, hedgehog theory can be seen as an attempt to extend certain parts of the Brunn-Minkowski theory to $\mathcal{H}^{n+1}$. For $n \leq 2$, it goes back to a paper by H. Geppert [3] who introduced hedgehogs under the German names stützbare Bereiche $(n=1)$ and stützbare Flächen $(n=2)$.

Let us recall the definition of hedgehogs with $C^{2}$ support functions in $\mathbb{R}^{n+1}$. For details on convex bodies, we refer the reader to the book by R. Schneider [22] As is well-known, every convex body $K \subset \mathbb{R}^{n+1}$ is determined by its support function $h_{K}: \mathbb{S}^{n} \longrightarrow \mathbb{R}$, where $h_{K}(u)$ is defined by $h_{K}(u)=\sup \{\langle x, u\rangle \mid x \in K\}$, $\left(u \in \mathbb{S}^{n}\right)$, that is, as the signed distance from the origin to the support hyperplane with unit normal vector $u$. In particular, every closed convex hypersurface of class $C_{+}^{2}$ (i.e., $C^{2}$-hypersurface with positive Gaussian curvature) is determined by its support function $h$ (which must be of class $C^{2}$ on $\mathbb{S}^{n}$ [22, p. 111]) as the envelope $\mathcal{H}_{h}$ of the family of hyperplanes with equation $\langle x, u\rangle=h(u)$. This envelope $\mathcal{H}_{h}$ is described analytically by the following system of equations

$$
\left\{\begin{array}{c}
\langle x, u\rangle=h(u) \\
\langle x, .\rangle=d h_{u}(.)
\end{array}\right.
$$

The second equation is obtained from the first by performing a partial differentiation with respect to $u$. From the first equation, the orthogonal projection of $x$ onto the line spanned by $u$ is $h(u) u$ and from the second one, the orthogonal projection of $x$ onto $u^{\perp}$ is the gradient of $h$ at $u$ (cf. Figure 3). Therefore, for each $u \in \mathbb{S}^{n}, x_{h}(u)=h(u) u+(\nabla h)(u)$ is the unique solution of this system. 


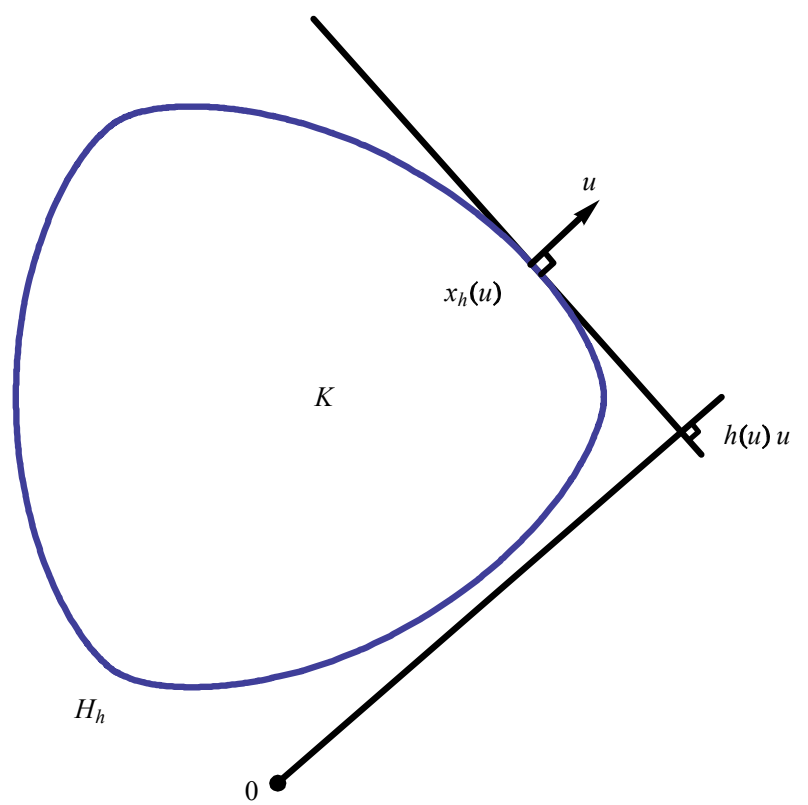

Figure 3. Convex bodies of class $C_{+}^{2}$ as envelopes parametrized by their Gauss map

Now, for any $C^{2}$ function $h$ on $\mathbb{S}^{n}$, the envelope $\mathcal{H}_{h}$ is in fact well-defined (even if $h$ is not the support function of a convex hypersurface). Its natural parametrization $x_{h}: \mathbb{S}^{n} \rightarrow \mathcal{H}_{h}, u \mapsto h(u) u+(\nabla h)(u)$ can be interpreted as the inverse of its Gauss map, in the sense that: at each regular point $x_{h}(u)$ of $\mathcal{H}_{h}$, $u$ is a normal vector to $\mathcal{H}_{h}$. We say that $\mathcal{H}_{h}$ is the hedgehog (or $C^{2}$-hedgehog) with support function $h$ (cf. Figure 4 ). Note that $x_{h}$ depends linearly on $h$.

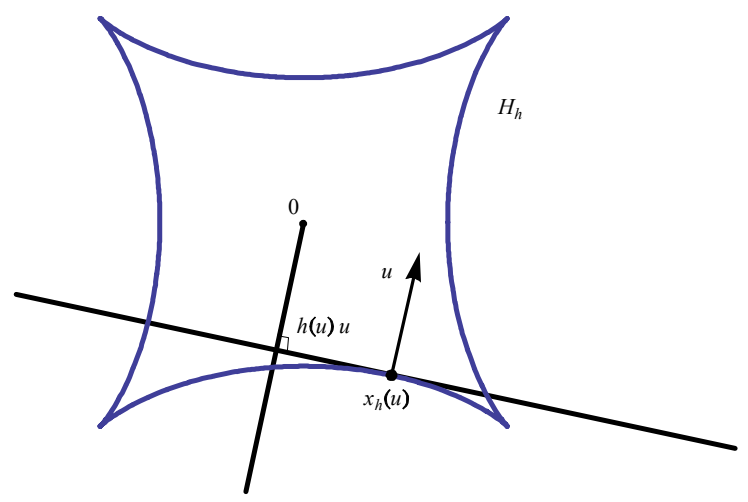

Figure 4. A $C^{2}$-hedgehog 
Since the parametrization $x_{h}$ can be regarded as the inverse of the Gauss map of $\mathcal{H}_{h}$, the Gaussian curvature $\kappa_{h}$ of $\mathcal{H}_{h}$ at $x_{h}(u)$ is given by $\kappa_{h}(u)=$ $1 / \operatorname{det}\left[T_{u} x_{h}\right]$, where $T_{u} x_{h}$ is the tangent map of $x_{h}$ at $u$. Therefore, singularities are the very points at which the Gaussian curvature is infinite. For every $u \in \mathbb{S}^{n}$, the tangent map of $x_{h}$ at the point $u$ is $T_{u} x_{h}=h(u) I d_{T_{u} \mathbb{S}^{n}}+H_{h}^{u}$, where $H_{h}^{u}$ is the symmetric endomorphism associated with the hessian of $h$ at $u$ [5]. Thus, the so-called 'curvature function' $R_{h}:=1 / \kappa_{h}$ is well-defined and continuous all over the unit sphere, including at the singular points (so that the classical Minkowski problem arises naturally for hedgehogs $[16,17])$.

Given a hedgehog $\mathcal{H}_{h} \subset \mathbb{R}^{n+1},(n \geq 1)$, the Kronecker index of $x \in \mathbb{R}^{n+1} \backslash \mathcal{H}_{h}$ with respect to $\mathcal{H}_{h}$, say $i_{h}(x)$, can be defined as the degree of the map

$$
\mathcal{U}_{(h, x)}: \mathbb{S}^{n} \rightarrow \mathbb{S}^{n}, u \longmapsto \frac{x_{h}(u)-x}{\left\|x_{h}(u)-x\right\|},
$$

and interpreted as the algebraic intersection number of an oriented half-line with origin $x$ with the hypersurface $\mathcal{H}_{h}$ equipped with its transverse orientation (number independent of the oriented half-line for an open dense set of directions) [5]. The (algebraic $(n+1)$-dimensional) volume of a hedgehog $\mathcal{H}_{h} \subset \mathbb{R}^{n+1}$ can be defined by

$$
v_{n+1}(h):=\int_{\mathbb{R}^{n+1} \backslash \mathcal{H}_{h}} i_{h}(x) d \lambda(x),
$$

where $\lambda$ denotes the Lebesgue measure on $\mathbb{R}^{n+1}$, and it satisfies

$$
v_{n+1}(h)=\frac{1}{n+1} \int_{\mathbb{S}^{n}} h(u) R_{h}(u) d \sigma(u),
$$

where $R_{h}$ is the curvature function and $\sigma$ the spherical Lebesgue measure on $\mathbb{S}^{n}$. As for convex bodies of class $C_{+}^{2}$, we introduce a mixed curvature function $R_{\left(f_{1}, \ldots, f_{n}\right)}$ and define the mixed (algebraic $(n+1)$-dimensional) volume of $n+1$ hedgehogs $\mathcal{H}_{h_{1}}, \ldots, \mathcal{H}_{h_{n+1}}$ of $\mathbb{R}^{n+1}$ by

$$
v_{n+1}\left(h_{1}, \ldots, h_{n+1}\right)=\frac{1}{n+1} \int_{\mathbb{S}^{n}} h_{1}(p) R_{\left(h_{2}, \ldots, h_{n+1}\right)}(p) d \sigma(p),
$$

where $R_{\left(h_{2}, \ldots, h_{n+1}\right)}$ denotes the mixed curvature function of $\mathcal{H}_{h_{2}}, \ldots, \mathcal{H}_{h_{n+1}}[10]$. See [7] for a study of this extension of the mixed volume and Alexandrov-Fenchel type inequalities for hedgehogs.

\section{Euler calculus}

Euler calculus is an integration theory built with the Euler characteristic $\chi$ as a finitely additive measure. Born in the sheaf theory, it has applications to algebraic topology, to stratified Morse theory, for reconstructing objects in 
integral geometry and for enumeration problems in computational geometry and sensor networks [2]. The short survey papers by P. Schapira [23] and O. Viro [25] played an important role in the development of this theory.

For the convenience of the reader, we briefly summarize in this section very basic notions and results from Euler calculus. For proofs and more information on Euler calculus and its applications, we refer the reader to [2].

Tame sets. In Euler calculus, the measurable sets are the tame sets in some fixed 0-minimal structure. We shall not recall here the definition of tame subsets in a fixed 0-minimal structure. It can be found in the classical surveys on Euler calculus, e.g., in [24]. Classical examples include polyconvex sets, semialgebraic sets and subanalytic sets. Here, we shall only need to know some basic facts that we shall summarize below. In particular, we shall need to know that the union and intersection of two tame sets are again tame.

Euler characteristic. Fix an 0 -minimal structure $\mathcal{O}$ on a topological space $X$. Definable functions between two spaces are those whose graphs are in $\mathcal{O}$. The Euler characteristic $\chi: \mathcal{O} \rightarrow \mathbb{Z}$ admits the following combinatorial definition:

Any tame set $A \in \mathcal{O}$ is definably homeomorphic to a finite disjoint union of open simplices $\coprod_{i} \sigma_{i}$ and we set:

$$
\chi(A)=\sum_{i}(-1)^{\operatorname{dim}\left(\sigma_{i}\right)} .
$$

Algebraic topology asserts that this quantity is well-defined, that is, independent of the decomposition into open simplices. This combinatorial Euler characteristic is a topological invariant. It is also a homotopy invariant for compact finite cell complexes (but not for non-compact spaces).

Examples. 1. Euler characteristic can be regarded as a generalization of cardinality. For a finite discrete tame set $A, \chi(A)$ is the cardinality of $A$ :

$$
\chi(A)=\# A
$$

2. A closed orientable 2-manifold $S$ has Euler characteristic $2-2 g$, where $g$ denotes the genus of $S$;

3. If $A$ is a compact contractible tame set, then $\chi(A)=1$;

4. Any open $n$-ball of $\mathbb{R}^{n}$ has Euler characteristic $(-1)^{n}$;

5. The $n$-dimensional sphere $\mathbb{S}^{n}$ has Euler characteristic $1+(-1)^{n}$;

6. The Euler characteristic of any odd-dimensional compact manifold is equal to zero (see [6] for an elementary proof).

Remarks. 1. Euler calculus relies on the following additivity property:

Proposition. For any pair $\{A, B\}$ of tame subsets of $X$, we have:

$$
\chi(A \cup B)=\chi(A)+\chi(B)-\chi(A \cap B) .
$$


2. Euler characteristic is multiplicative under cross products:

Proposition. For any pair $\{E, F\}$ of tame sets, we have:

$$
\chi(E \times F)=\chi(E) \cdot \chi(F) .
$$

Note that these additivity and multiplicativity properties generalize the ones of cardinality of sets.

Euler integral. The above additivity property suggests to define a measure over tame sets via:

$$
\int_{X} \mathbf{1}_{A}(x) d \chi=\chi(A)
$$

where $\mathbf{1}_{A}$ is the characteristic function over a tame subset $A$ of $X$. A function $f: X \rightarrow \mathbb{Z}$ is said to be constructible if it has finite range and if all its level sets $f^{-1}(\{s\})$ are tame subsets of $X$. Let $C F(X)$ denote the $\mathbb{Z}$-module of all $\mathbb{Z}$-valued constructible functions on $X$. The Euler integral is defined to be the homomorphism $\int_{X}: C F(X) \rightarrow \mathbb{Z}$ given by:

$$
\int_{X} f d \chi:=\sum_{s=-\infty}^{+\infty} s \chi\left[f^{-1}(\{s\})\right] .
$$

Alternately, writing $f \in C F(X)$ as $f=\sum_{i} c_{i} \mathbf{1}_{\sigma_{i}}$, where $X=\coprod_{i} \sigma_{i}$ is a decomposition of $X$ into a finite disjoint union of open cells and where $c_{i} \in \mathbb{Z}$, we have:

$$
\int_{X} f d \chi:=\sum_{i} c_{i} \chi\left(\sigma_{i}\right)=\sum_{i} c_{i}(-1)^{\operatorname{dim}\left(\sigma_{i}\right)}
$$

Convolution. On a finite-dimensional real vector space $V$, a convolution operator with respect to Euler characteristic is defined as follows:

$$
\forall(f, g) \in C F(V)^{2}, \quad(f * g)(x)=\int_{V} f(y) g(x-y) d y .
$$

Convolution is a commutative, associative operator providing $C F(V)$ with the structure of an algebra.

Proposition. $(C F(V),+, *)$ is a commutative ring with multiplicative identity element $\mathbf{1}_{\left\{0_{\mathrm{v}}\right\}}$. 
Relationship with Minkowski addition. There is a close relationship between Minkowski addition and convolution with respect to the Euler characteristic $[4,23,25]$ :

Theorem [4]. Let $A$ and $B$ be two compact convex subsets of $\mathbb{R}^{n+1}$. We have

$$
\mathbf{1}_{A} * \mathbf{1}_{B}=\mathbf{1}_{A+B},
$$

where $*$ denotes the convolution product with respect to the Euler characteristic and $A+B$ the usual Minkowski sum of $A$ and $B$.

This relationship will be the starting point in our study.

\section{Main results}

In this section, given a convex body $K \subset \mathbb{R}^{n+1}$, we shall often need $\stackrel{o}{K}$ and $\partial K$ to be tame subsets of $\mathbb{R}^{n+1}$. It is the reason why we shall restrict ourselves to analytic hedgehogs (resp. convex bodies).

\section{Minkowski inversion with respect to $\chi$}

Since $\mathbf{1}_{\left\{0_{\mathbb{R}^{n+1}}\right\}}$ is the multiplicative identity of $\left(C F\left(\mathbb{R}^{n+1}\right),+, *\right)$, the following result can be regarded as a Minkowski inversion theorem:

Theorem 1 Let $K \subset \mathbb{R}^{n+1}$ be a convex body of class $C_{+}^{\omega}$. We have

$$
(-1)^{n+1}\left(\mathbf{1}_{K} * \mathbf{1}_{-K}^{\circ}\right)=\mathbf{1}_{\left\{0_{\mathbb{R}^{n+1}}\right\}},
$$

where $-\stackrel{o}{K}$ denotes the reflection of $\stackrel{o}{K}$ through the origin $0_{\mathbb{R}^{n+1}}$. In other words, the convolution inverse of the characteristic function of $K$ is given by:

$$
\left(\mathbf{1}_{K}\right)^{-1}=(-1)^{n+1} \mathbf{1}_{-K}^{o} .
$$

Remarks. 1. Of course, if $K$ is a convex body reduced to a point $a$ of $\mathbb{R}^{n+1}$, then the convolution inverse of the characteristic function of $K$ is given by:

$$
\left(\mathbf{1}_{K}\right)^{-1}=\mathbf{1}_{\{-a\}} .
$$

2. In [20], Pukhlikov and Khovanskii gave a similar Minkowski inversion theorem in the polytopal case: for every convex polytope $K \subset \mathbb{R}^{n+1}$, we have

$$
(-1)^{\operatorname{dim} K}\left(\mathbf{1}_{K} * \mathbf{1}_{- \text {relint } K}\right)=\mathbf{1}_{\left\{0_{\mathbb{R}^{n+1}}\right\}},
$$

where relint $K$ is the relative interior of $K$, that is, the interior of $K$ in the smallest affine subspace that contains $K$. 


\section{Euler index}

Definition Let $\mathcal{H}_{h}$ be a $C^{\omega}$-hedgehog of $\mathbb{R}^{n+1}$ and let $K, L \subset \mathbb{R}^{n+1}$ be convex bodies of class $C_{+}^{\omega}$ such that $\mathcal{H}_{h}$ is representing the formal difference $K-L$. Define the Euler index of $\mathcal{H}_{h}$ by

$$
\mathbf{1}_{h}:=\mathbf{1}_{K} *\left(\mathbf{1}_{L}\right)^{-1}=(-1)^{n+1}\left(\mathbf{1}_{K} * \mathbf{1}_{-L}\right),
$$

where $-\stackrel{o}{L}$ denotes the reflection of $\stackrel{o}{L}$ through the origin $0_{\mathbb{R}^{n+1}}$.

Remarks. 1. Given any $C^{\omega}$-hedgehog $\mathcal{H}_{h} \subset \mathbb{R}^{n+1}$, for every large enough $r>0, k:=h+r$ and $l:=r$ are the respective support functions of two convex bodies $K$ and $L$ such that $\mathcal{H}_{h}$ is representing the formal difference $K-L$.

2. Using Groemer's theorem and properties of convolution product, we can easily check that $\mathbf{1}_{h}$ is independent of the choice of the pair $(K, L)$ of convex bodies of class $C_{+}^{\omega}$ such that $\mathcal{H}_{h}$ is representing $K-L$.

Furthermore, Groemer's theorem admits the following extension to analytic hedgehogs:

Theorem 2 Let $\mathcal{H}_{f}$ and $\mathcal{H}_{g}$ be two analytic hedgehogs of $\mathbb{R}^{n+1}$. We have

$$
\mathbf{1}_{f} * \mathbf{1}_{g}=\mathbf{1}_{f+g} .
$$

This can be easily deduced from Groemer's theorem by using the above Minkowski inversion theorem.

\section{Relationship with Kronecker index}

Theorem 3 Let $\mathcal{H}_{h}$ be a $C^{\omega}$-hedgehog of $\mathbb{R}^{n+1}$ and let $K, L \subset \mathbb{R}^{n+1}$ be convex bodies of class $C_{+}^{\omega}$ such that $\mathcal{H}_{h}$ is representing the formal difference $K-L$.

For any $x \in \mathbb{R}^{n+1} \backslash \mathcal{H}_{h}$, the Euler index $\mathbf{1}_{h}(x):=(-1)^{n+1}\left(\mathbf{1}_{K} * \mathbf{1}_{-L}\right)(x)$ of $\mathcal{H}_{h}$ at $x$ is equal to $i_{h}(x)$, that is, to the degree of the map

$$
\mathcal{U}_{(h, x)}: \mathbb{S}^{n} \rightarrow \mathbb{S}^{n}, u \longmapsto \frac{x_{h}(u)-x}{\left\|x_{h}(u)-x\right\|} .
$$

In other words, the Kronecker index $i_{h}$ is nothing but the restriction of the Euler index to $\mathbb{R}^{n+1} \backslash \mathcal{H}_{h}$.

\section{Expressions for the Kronecker index}

Theorem 4 Let $\mathcal{H}_{h} \subset \mathbb{R}^{n+1}$ be a $C^{\omega}$-hedgehog. Fix $x \in \mathbb{R}^{n+1} \backslash \mathcal{H}_{h}$ and let $h_{x}: \mathbb{S}^{n} \rightarrow \mathbb{R}$ be the support function of $\mathcal{H}_{h}$ with respect to $x$ :

$$
h_{x}(u):=\left\langle x_{h}(u)-x, u\right\rangle=h(u)-\langle x, u\rangle .
$$


The Kronecker index $i_{h}(x)$ is given by

$$
i_{h}(x)=1+(-1)^{n+1} \chi_{h}^{-}(x)=\chi_{h}^{+}(x)+(-1)^{n+1},
$$

where $\chi_{h}^{-}(x):=\chi\left[\left(h_{x}\right)^{-1}(]-\infty, 0[)\right]$ and $\chi_{h}^{+}(x):=\chi\left[\left(h_{x}\right)^{-1}(] 0,+\infty[)\right]$.

Corollary 5 Under the assumptions of the previous theorem, we have:

$$
\forall x \in \mathbb{R}^{n+1} \backslash \mathcal{H}_{h}, \quad i_{h}(x)= \begin{cases}1-\frac{1}{2} \chi_{h}(x) & \text { if } n+1 \text { is even } \\ \frac{1}{2}\left(\chi_{h}^{+}(x)-\chi_{h}^{-}(x)\right) & \text { if } n+1 \text { is odd },\end{cases}
$$

where $\chi_{h}(x):=\chi\left[\left(h_{x}\right)^{-1}(\{0\})\right], \chi_{h}^{-}(x):=\chi\left[\left(h_{x}\right)^{-1}(]-\infty, 0[)\right]$ and $\chi_{h}^{+}(x):=$ $\chi\left[\left(h_{x}\right)^{-1}(] 0,+\infty[)\right]$.

Remarks. 1. From these results, if $n+1$ is even then, for any $x \in \mathbb{R}^{n+1} \backslash \mathcal{H}_{h}$, the knowledge of one of the four integers $\chi_{h}(x), \chi_{h}^{-}(x), \chi_{h}^{+}(x)$ and $i_{h}(x)$ implies that of the three others.

2. For $n+1=2$, we proved the following more general result (recall that the Euler characteristic is a generalization of cardinality):

Theorem [8]. Let $\mathcal{H}_{h} \subset \mathbb{R}^{2}$ be a $C^{2}$-hedgehog. For every $x \in \mathbb{R}^{2} \backslash \mathcal{H}_{h}$, the Kronecker index $i_{h}(x)$ is given by

$$
i_{h}(x)=1-\frac{1}{2} n_{h}(x),
$$

where $n_{h}(x)$ denotes the number of cooriented support lines of $\mathcal{H}_{h}$ through $x$, that is, the number of zeros of $h_{x}: \mathbb{S}^{1} \rightarrow \mathbb{R}, u \mapsto h(u)-\langle x, u\rangle$.

3. For $n+1=3$, another expression for $i_{h}(x)$ is given by:

Theorem [16]. Let $\mathcal{H}_{h} \subset \mathbb{R}^{3}$ be a $C^{2}$-hedgehog. For every $x \in \mathbb{R}^{3} \backslash \mathcal{H}_{h}$, the Kronecker index $i_{h}(x)$ is given by

$$
i_{h}(x)=r_{h}^{+}(x)-r_{h}^{-}(x),
$$

where $r_{h}^{-}(x)$ (resp. $\left.r_{h}^{+}(x)\right)$ denotes the number of connected components of $\mathbb{S}^{2}-h_{x}^{-1}(\{0\})$ on which $h_{x}(u):=h(u)-\langle x, u\rangle$ is negative (resp. positive).

4. In [16], we introduced another notion of index, which is more appropriate for studying certain classes of hedgehogs of $\mathbb{R}^{3}$, such as projective hedgehogs (i.e. with an antisymmetric support function): 
Definition. Let $\mathcal{H}_{h} \subset \mathbb{R}^{3}$ be a $C^{2}$-hedgehog. Fix $x \in \mathbb{R}^{3} \backslash \mathcal{H}_{h}$ and let $h_{x}$ : $\mathbb{S}^{2} \rightarrow \mathbb{R}$ be the support function of $\mathcal{H}_{h}$ with respect to $x$. For every $x \in \mathbb{R}^{3}-\mathcal{H}_{h}$, define the $j_{h}$-index of $x$ with respect to $\mathcal{H}_{h}$ by:

$$
j_{h}(x):=1-c_{h}(x),
$$

where $c_{h}(x)$ denotes the number of connected components of $h_{x}^{-1}(\{0\}) \subset \mathbb{S}^{2}$, that is the number of spherical curves corresponding to points of $\mathcal{H}_{h}$ at which the support plane passes through $x$.

Euler index at a point of $\mathcal{H}_{h} \subset \mathbb{R}^{2}$

Theorem 6 Let $\mathcal{H}_{h} \subset \mathbb{R}^{2}$ be a $C^{\omega}$-hedgehog. At a simple regular point $x:=$ $x_{h}(u)$ of $\mathcal{H}_{h}$, the Euler index $\mathbf{1}_{h}(x)$ is equal to the value taken by the Kronecker index $i_{h}$ on the connected component of $\mathbb{R}^{2} \backslash \mathcal{H}_{h}$ towards which the unit normal vector $-u$ is pointing to. At a simple cusp point c of $\mathcal{H}_{h}$, the Euler index $\mathbf{1}_{h}(c)$ is equal to the value taken by the Kronecker index $i_{h}$ on the connected component of $\mathbb{R}^{2} \backslash \mathcal{H}_{h}$ that lies, in a neighborhood $\Omega$ of $c$, on the same side of $\mathcal{H}_{h}$ as the evolute of $\mathcal{H}_{h} \cap \Omega$.

Remarks. 1. Generic singularities of plane $C^{\infty}$-hedgehogs are cusp points [5].

2. This result can be extended to hedgehogs $\mathcal{H}_{h} \subset \mathbb{R}^{2}$ that are Minkowski differences $K-L$ of convex polygons (see Figure 5).
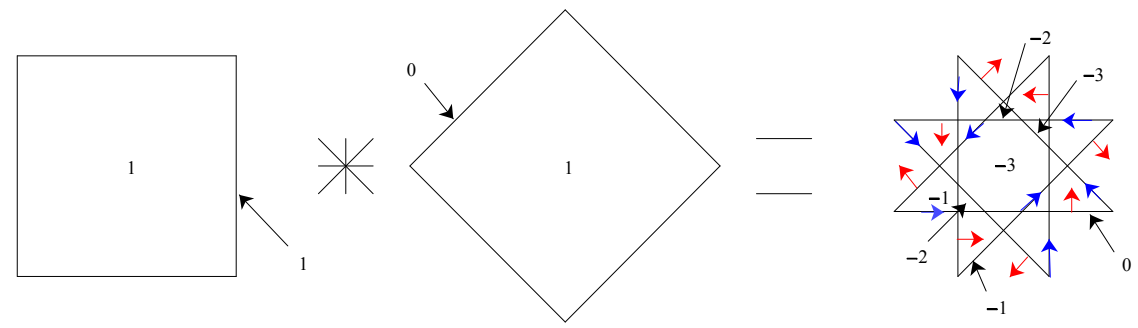

Figure 5. Euler index of the Minkowski difference of two squares

\section{Euler index at a regular point of $\mathcal{H}_{h} \subset \mathbb{R}^{n+1}$}

In higher dimensions, the question is more involved at the singular points. However, the result remains true at the simple regular points.

Theorem 7 Let $\mathcal{H}_{h} \subset \mathbb{R}^{n+1}$ be a $C^{\omega}$-hedgehog. At a simple regular point $x:=x_{h}(u)$ of $\mathcal{H}_{h}$, the Euler index $\mathbf{1}_{h}(x)$ is equal to the value taken by the Kronecker index $i_{h}$ on the connected component of $\mathbb{R}^{n+1} \backslash \mathcal{H}_{h}$ towards which the unit normal vector $-u$ is pointing to. 


\section{Proof of the results}

Proof of Theorem 1. By the definition of the convolution product, we have

$$
\left(\mathbf{1}_{K} * \mathbf{1}_{-K}\right)(x):=\int_{\mathbb{R}^{n+1}} \mathbf{1}_{K}(y) \mathbf{1}_{-K}^{o}(x-y) d \chi(y) \quad \text { for } x \in \mathbb{R}^{n+1} .
$$

Fix $x \in \mathbb{R}^{n+1}$. The range of $F_{x}: \mathbb{R}^{n+1} \rightarrow \mathbb{R}, y \longmapsto \mathbf{1}_{K}(y) \mathbf{1}_{-K}^{o}(x-y)$ is included in $\{0,1\}$ and

$$
\forall y \in \mathbb{R}^{n+1}, \quad F_{x}(y)=1 \Leftrightarrow y \in K \cap(\stackrel{o}{K}+\{x\}) .
$$

By the definition of Euler integral, we thus get

$$
\begin{gathered}
\quad\left(\mathbf{1}_{K} * \mathbf{1}_{-K}^{o}\right)(x):=\int_{\mathbb{R}^{n+1}} F_{x}(y) d \chi(y)=\chi[K \cap(\stackrel{o}{K}+\{x\})] . \\
\text { If } x=0_{\mathbb{R}^{n+1}} \text { then } K \cap(\stackrel{o}{K}+\{x\})=\stackrel{o}{K} \text { and hence }\left(\mathbf{1}_{K} * \mathbf{1}_{-K}^{o}\right)(x)=(-1)^{n+1}
\end{gathered}
$$
since $\stackrel{o}{K}$ is homeomorphic to an open $(n+1)$-ball.

Assume $x \neq 0_{\mathbb{R}^{n+1}}$. If $K \cap(\stackrel{o}{K}+\{x\})=\varnothing$ then $\chi[K \cap(\stackrel{o}{K}+\{x\})]=0$. Hence, we may assume that $K \cap(\stackrel{o}{K}+\{x\}) \neq \varnothing$. In this case, $\stackrel{\circ}{K} \cap(\stackrel{\circ}{K}+\{x\})$ is homeomorphic to an open $(n+1)$-ball and its boundary is the disjoint union of $\partial K \cap(\stackrel{o}{K}+\{x\})$ and $K \cap \partial(K+\{x\})$, where the boundary of a convex body $L$ is denoted by $\partial L$. Therefore, $K \cap(\stackrel{o}{K}+\{x\})$ is then the disjoint union of $\stackrel{o}{K} \cap(\stackrel{o}{K}+\{x\})$ and $\partial K \cap(\stackrel{o}{K}+\{x\})$, which is homeomorphic to an open $n$-ball, so that

$$
\begin{aligned}
\chi[K \cap(\stackrel{o}{K}+\{x\})] & =\chi[\stackrel{o}{K} \cap(\stackrel{o}{K}+\{x\})]+\chi[\partial K \cap(\stackrel{o}{K}+\{x\})] \\
& =(-1)^{n+1}+(-1)^{n} \\
& =0,
\end{aligned}
$$

which achieves the proof.

To prove Theorems 3 and 4, we shall need some intermediate results and properties. 
Proposition 8 Under assumptions of Theorem 3, we have:

$$
\mathbf{1}_{h}(x)= \begin{cases}=i_{h}(x)=0 & \text { if } x \notin K+(-L) \\ (-1)^{n+1}(1-\chi[(K+\{-x\}) \cap \partial L]) & \text { if } x \in(K+(-L)) \backslash \mathcal{H}_{h} .\end{cases}
$$

Proof. We have

$$
\mathbf{1}_{K} * \mathbf{1}_{-L}=\mathbf{1}_{K} *\left(\mathbf{1}_{-L}-\mathbf{1}_{-\partial L}\right)=\left(\mathbf{1}_{K} * \mathbf{1}_{-L}\right)-\left(\mathbf{1}_{K} * \mathbf{1}_{-\partial L}\right),
$$

where $-L$ (resp. $-\partial L$ ) denotes the reflection of $L$ (resp. $\partial L$ ) through the origin. Now, we have $\mathbf{1}_{K} * \mathbf{1}_{-L}=\mathbf{1}_{K+(-L)}$ by Groemer's theorem, so that

$$
\mathbf{1}_{K} * \mathbf{1}_{-L}^{o}=\mathbf{1}_{K+(-L)}-\left(\mathbf{1}_{K} * \mathbf{1}_{-\partial L}\right) .
$$

Let $x \in \mathbb{R}^{n+1}$. The range of $F_{x}: \mathbb{R}^{n+1} \rightarrow \mathbb{R}, y \longmapsto \mathbf{1}_{K}(y) \mathbf{1}_{-\partial L}(x-y)$ is included in $\{0,1\}$ and

$$
\forall y \in \mathbb{R}^{n+1}, \quad F_{x}(y)=1 \Leftrightarrow y \in K \cap(\partial L+\{x\}) .
$$

By the definition of Euler integral, we thus get

$$
\left(\mathbf{1}_{K} * \mathbf{1}_{-\partial L}\right)(x):=\int_{\mathbb{R}^{n+1}} F_{x}(y) d \chi(y)=\chi[K \cap(\partial L+\{x\})] .
$$

Using the translation $y \mapsto y-x$, we deduce that

$$
\left(\mathbf{1}_{K} * \mathbf{1}_{-\partial L}\right)(x)=\chi[(K+\{-x\}) \cap \partial L] .
$$

First assume $x \notin K+(-L)$. Then $\mathbf{1}_{K+(-L)}(x)=0$ and $\left(\mathbf{1}_{K} * \mathbf{1}_{-\partial L}\right)(x)=0$ since $(K+\{-x\}) \cap \partial L \neq \varnothing$ would imply $x \in K+(-\partial L)$. Consequently

$$
\begin{aligned}
\mathbf{1}_{h}(x) & :=(-1)^{n+1}\left(\mathbf{1}_{K} * \mathbf{1}_{-L}^{o}\right)(x) \\
& =(-1)^{n+1}\left(\mathbf{1}_{K+(-L)}(x)-\left(\mathbf{1}_{K} * \mathbf{1}_{-\partial L}\right)(x)\right) \\
& =0 .
\end{aligned}
$$

Since $x_{h}\left(\mathbb{S}^{n}\right) \subset K+(-L)$, we also have $i_{h}(x)=0$ and thus $\mathbf{1}_{h}(x)=i_{h}(x)$.

Now assume $x \in(K+(-L)) \backslash \mathcal{H}_{h}$. Then we get

$$
\mathbf{1}_{h}(x)=(-1)^{n+1}(1-\chi[(K+\{-x\}) \cap \partial L]) .
$$

Recall that we say that two submanifolds $S_{1}$ and $S_{2}$ of a manifold $M$ are transverse, and we write $S_{1} \pitchfork S_{2}$, if $T_{m} M=T_{m} S_{1}+T_{m} S_{2}$ for all $m \in S_{1} \cap S_{2}$. 
Proposition 9 Let $\mathcal{H}_{h}$ be a $C^{\omega}$-hedgehog of $\mathbb{R}^{n+1}$ and let $K, L \subset \mathbb{R}^{n+1}$ be convex bodies of class $C_{+}^{\omega}$ such that $\mathcal{H}_{h}$ is representing the formal difference $K-L$. For every $x \in \mathbb{R}^{n+1}$ such that $(K+\{-x\}) \cap L \neq \varnothing$ and $\partial(K+\{-x\}) \pitchfork \partial L$, the following properties hold:

(i) $\left(h_{x}\right)^{-1}(\{0\}) \approx \partial(K+\{-x\}) \cap \partial L$;

(ii) $\left.\left.\left(h_{x}\right)^{-1}(]-\infty, 0\right]\right) \approx \partial(K+\{-x\}) \cap L$;

(iii) $\left(h_{x}\right)^{-1}([0,+\infty[) \approx(K+\{-x\}) \cap \partial L$;

where $\approx$ is the homeomorphism relation and $\left(h_{x}\right)(u):=h(u)-\langle x, u\rangle,\left(u \in \mathbb{S}^{n}\right)$.

Proof. (i) It follows from the assumptions that $(K+\{-x\}) \cap L$ is a strictly convex body with interior points, and thus that its support function

$$
\begin{aligned}
f: & \mathbb{S}^{n} \longrightarrow \mathbb{R} \\
& u \mapsto \sup \{\langle p, u\rangle \mid p \in(K+\{-x\}) \cap L\}
\end{aligned}
$$

is continuously differentiable (see, e.g., [22]). Denote by $k$ and $l$ the respective support functions of $K$ and $L$ and let $k_{x}(u):=k(u)-\langle x, u\rangle$ for all $u \in \mathbb{S}^{n}$. Note that the zeros of $h_{x}=k_{x}-l$ are the points $u \in \mathbb{S}^{n}$ such that the support hyperplanes with exterior normal vector $u$ of $K+\{-x\}$ and $L$ coincide. Such an $u \in\left(h_{x}\right)^{-1}(\{0\})$ cannot be a regular point of $x_{f}$. So, we can consider the continuous map

$$
\begin{aligned}
\phi: & \left(h_{x}\right)^{-1}(\{0\}) \rightarrow \partial(K+\{-x\}) \cap \partial L \\
& u \longmapsto x_{f}(u):=(\nabla f)(u)+f(u) u
\end{aligned}
$$

To check that it defines a homeomorphism from the compact $\left(h_{x}\right)^{-1}(\{0\})$ to $\partial(K+\{-x\}) \cap \partial L$, it suffices to prove that it is a bijection.

Let $p \in \partial(K+\{-x\}) \cap \partial L$. Since $\partial(K+\{-x\}) \pitchfork \partial L$, there exists a pair of non-antipodal points $v$ and $w$ on $\mathbb{S}^{n}$, such that

$$
p=x_{k_{x}}(v)=x_{l}(w) .
$$

Let $\gamma$ denote the shortest arc between $v$ and $w$ on $\mathbb{S}^{n}$. Since we have clearly $h_{x}(v)<0$ and $h_{x}(w)>0$, there exists some $u \in \gamma$ such that $h_{x}(u)=0$. It remains to prove that such an $u \in \gamma$ is unique and such that $\phi(u)=p$. For $\xi \in \mathbb{S}^{n}$, let $H_{k_{x}}(\xi)$ and $H_{l}(\xi)$ (resp. $H_{k_{x}}^{-}(\xi)$ and $H_{l}^{-}(\xi)$ ) denote the respective support hyperplanes (resp. halfspaces) with exterior normal vector $\xi$ of $K+\{-x\}$ and $L$. Note that: $(\alpha)$ The segment with endpoints $x_{k_{x}}(u)$ and $x_{l}(u)$, say $\sigma(u)$, is passing through the complementary of $H_{k_{x}}^{-}(v) \cup H_{l}^{-}(w) ;(\beta)$ $H_{k_{x}}(u)=H_{l}(u)=\left(x_{k_{x}}(u) x_{l}(u)\right)+\left(v^{\perp} \cap w^{\perp}\right)$, where $\xi^{\perp}$ is the vector subspace orthogonal to $\xi \in \mathbb{S}^{n}$ and $\left(x_{k_{x}}(u) x_{l}(u)\right)$ the line through $x_{k_{x}}(u)$ and $x_{l}(u)$.

Let $u_{1}, u_{2} \in \gamma \cap\left(h_{x}\right)^{-1}(\{0\})$. From $(\alpha)$ and $(\beta)$ with $u=u_{1}$ and $u=u_{2}$, it follows that the support hyperplanes $H_{k_{x}}\left(u_{1}\right)=H_{l}\left(u_{1}\right)$ and $H_{k_{x}}\left(u_{2}\right)=H_{l}\left(u_{2}\right)$ of the convex hull of $(K+\{-x\}) \cup L$ must coincide (in order that all the endpoints of the segments $\sigma\left(u_{1}\right)$ and $\sigma\left(u_{2}\right)$ lie in each of the support halfspaces $H_{l}^{-}\left(u_{1}\right)$ and $H_{l}^{-}\left(u_{2}\right)$, see Figure 6$)$. Therefore, there exists a unique $u \in \gamma$ 
such that $h_{x}(u)=0$ and it satisfies $\phi(u)=p$.

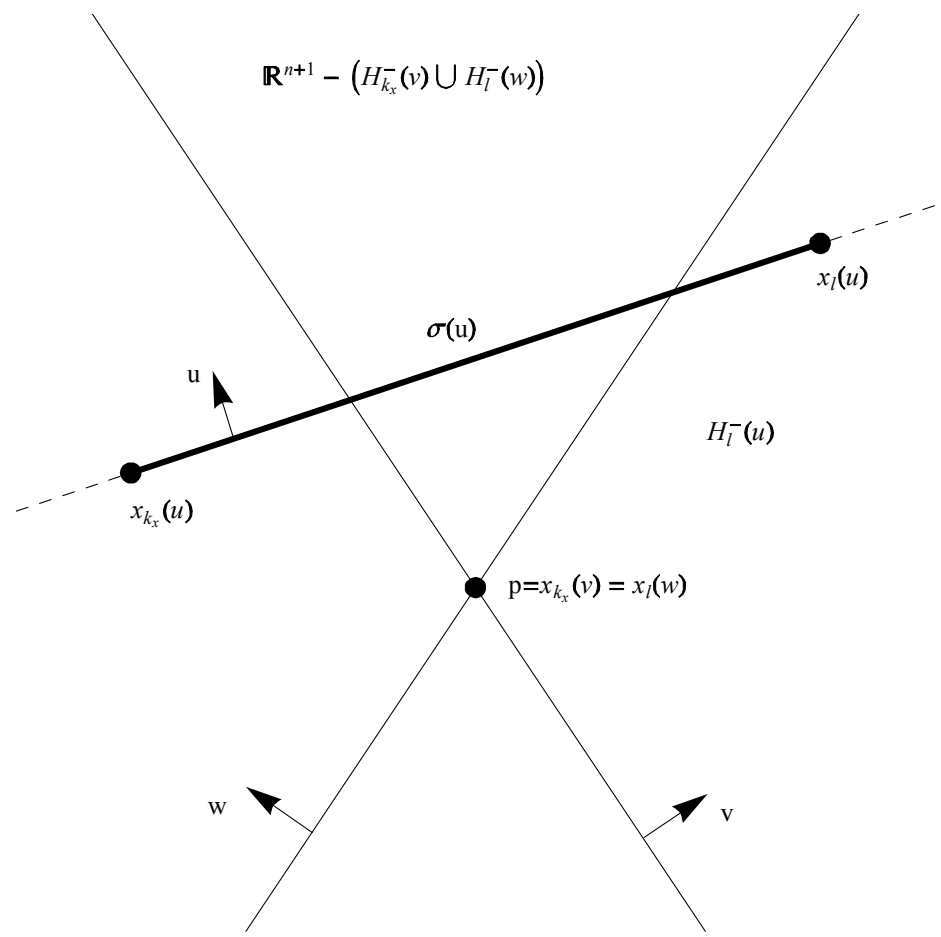

Figure 6. Projection view onto the plane $(\mathbb{R} v+\mathbb{R} w)^{\perp}$

The following corollary is immediate.

Corollary 10 Under the assumptions of the previous proposition, we have:

$$
\left\{\begin{array}{l}
\chi[\partial(K+\{-x\}) \cap \partial L]=\chi_{h}(x) \\
\chi[\partial(K+\{-x\}) \cap L]=\chi_{h}^{-}(x)+\chi_{h}(x) \\
\chi[(K+\{-x\}) \cap \partial L]=\chi_{h}(x)+\chi_{h}^{+}(x)
\end{array}\right.
$$

where $\chi_{h}(x):=\chi\left[\left(h_{x}\right)^{-1}(\{0\})\right], \chi_{h}^{-}(x):=\chi\left[\left(h_{x}\right)^{-1}(]-\infty, 0[)\right]$ and $\chi_{h}^{+}(x):=$ $\chi\left[\left(h_{x}\right)^{-1}(] 0,+\infty[)\right]$.

Lemma 11 Under assumptions of Theorem 3, we have:

$$
\mathbf{1}_{h}(x)=i_{h}(x)=1-(-1)^{n} \chi_{h}^{-}(x)
$$

if $\partial(K+\{-x\})$ and $\partial L$ are externally tangent. 
Proof. Let $a=b-x$ be the point of tangency of $\partial(K+\{-x\})$ and $\partial L$, where $(a, b) \in K \times L$. By Proposition 8, we have

$$
\mathbf{1}_{h}(x)=(-1)^{n+1}(1-\chi[(K+\{-x\}) \cap \partial L]) .
$$

Since $(K+\{-x\}) \cap \partial L=\{a\}$, this implies $\mathbf{1}_{h}(x)=0$.

Let $u$ be the point of $\mathbb{S}^{n}$ such that $a=x_{k_{x}}(u)=x_{l}(-u)$. For all $\varepsilon>0$, $x_{\varepsilon}:=x+\varepsilon u$ is such that $\left(K+\left\{-x_{\varepsilon}\right\}\right) \cap L=\varnothing$ and hence $x_{\varepsilon} \notin K+(-L)$. Therefore, $i_{h}\left(x_{\varepsilon}\right)=0$ for all $\varepsilon>0$ and hence $i_{h}(x)=0$.

Finally, by noticing that $\chi_{h}^{-}(x)$ is constant on each connected component of $\mathbb{R}^{n+1}-\mathcal{H}_{h}$ and that $\left(h_{x}\right)^{-1}(]-\infty, 0[)$ is homeomorphic to an open $n$-ball $B_{n}$ when the Euclidean norm of $x$ is sufficiently large, we see that

$$
\chi_{h}^{-}(x)=\chi\left(B_{n}\right)=(-1)^{n},
$$

which achieves the proof.

Lemma 12 Let $\mathcal{H}_{h} \subset \mathbb{R}^{n+1}$ be an analytic hedgehog. For every $x \in \mathbb{R}^{n+1} \backslash \mathcal{H}_{h}$, the index $\mathbf{1}_{h}(x)$ is given by

$$
\mathbf{1}_{h}(x)=1+(-1)^{n+1} \chi_{h}^{-}(x),
$$

where $\chi_{h}^{-}(x):=\chi\left[\left(h_{x}\right)^{-1}(]-\infty, 0[)\right]$.

Proof. From Proposition 8 and Lemma 11, we can assume without loss of generality that $x \in(K+(-L)) \backslash \mathcal{H}_{h}$ and $\partial(K+\{-x\}) \pitchfork \partial L$. Then, by Proposition 8 and Corollary 10, we have:

$$
\mathbf{1}_{h}(x)=(-1)^{n+1}\left(1-\left(\chi_{h}(x)+\chi_{h}^{+}(x)\right)\right) .
$$

But

$$
\chi_{h}^{-}(x)+\chi_{h}(x)+\chi_{h}^{+}(x)=\chi\left(\mathbb{S}^{n}\right) \quad \text { and } \quad \chi\left(\mathbb{S}^{n}\right)=1+(-1)^{n},
$$

so that

$$
\mathbf{1}_{h}(x)=1+(-1)^{n+1} \chi_{h}^{-}(x)
$$

Proof of Theorems 3 and 4 . Let $\mathcal{H}_{\widetilde{h}} \subset \mathbb{R}^{n+1}$ be the hedgehog with support function $\widetilde{h}(-u)=-h(u),\left(u \in \mathbb{S}^{n}\right)$. Note that $\mathcal{H}_{h}$ and $\mathcal{H}_{\widetilde{h}}$ have:

- the same geometric realization since $x_{\widetilde{h}}(-u)=x_{h}(u)$ for all $u \in \mathbb{S}^{n}$;

- the same transverse orientation (resp. opposite transverse orientations) at each point $x_{\widetilde{h}}(-u)=x_{h}(u)$ if $n+1$ is even (resp. odd).

Therefore $i_{\widetilde{h}}=(-1)^{n+1} i_{h}$ on $\mathbb{R}^{n+1} \backslash \mathcal{H}_{h}$. Thus if we prove that, under assumptions of Theorem $3, i_{h}(x)=\chi_{h}^{+}(x)+(-1)^{n+1}$ for all $x \in \mathbb{R}^{n+1} \backslash \mathcal{H}_{h}$, then 


$$
\begin{aligned}
i_{h}(x) & =(-1)^{n+1} i_{\widetilde{h}}(x) \\
& =(-1)^{n+1}\left(\chi_{\widetilde{h}}^{+}(x)+(-1)^{n+1}\right) \\
& =1+(-1)^{n+1} \chi_{\widetilde{h}}^{+}(x) \\
& =1+(-1)^{n+1} \chi_{h}^{-}(x) \quad \text { for all } x \in \mathbb{R}^{n+1} \backslash \mathcal{H}_{h},
\end{aligned}
$$

and hence $i_{h}=\mathbf{1}_{h}$ on $\mathbb{R}^{n+1} \backslash \mathcal{H}_{h}$ by Lemma 12 . So it remains only to prove that:

$$
\forall x \in \mathbb{R}^{n+1} \backslash \mathcal{H}_{h}, \quad i_{h}(x)=\chi_{h}^{+}(x)+(-1)^{n+1} .
$$

Since $i_{h}(x)$ is equal to 0 and $\left(h_{x}\right)^{-1}(] 0,+\infty[)$ homeomorphic to an open $n$ ball when the distance of $x$ from the origin is sufficiently large, it suffices to prove that the map $x \mapsto i_{h}(x)-\left(\chi_{h}^{+}(x)+(-1)^{n+1}\right)$ is constant on $\mathbb{R}^{n+1}-\mathcal{H}_{h}$. Since the maps $x \mapsto i_{h}(x)$ and $x \mapsto \chi_{h}^{+}(x)$ are constant on each connected component of $\mathbb{R}^{n+1}-\mathcal{H}_{h}$, we only need to prove that $i_{h}(x)-\chi_{h}^{+}(x)$ remains constant whenever $x$ crosses $\mathcal{H}_{h}$ transversally at a regular point.

Recall that, at a regular point $x_{h}(u)$ of $\mathcal{H}_{h}$, the transverse orientation of $\mathcal{H}_{h}$ is given by $\operatorname{sign}\left[R_{h}(u)\right] u$, where sign is the sign function and $R_{h}$ the curvature function of $\mathcal{H}_{h}$. Therefore, the Kronecker index $i_{h}(x)$ decreases by one unit whenever $x$ crosses $\mathcal{H}_{h}$ transversally at a simple regular point $x_{h}(u)$ in the direction of $\operatorname{sign}\left[R_{h}(u)\right] u$. Thus it is sufficient to prove that $\chi_{h}^{+}(x)$ also decreases by one unit whenever $x$ crosses $\mathcal{H}_{h}$ transversally at a simple regular point $x_{h}(u)$ in the direction of $\operatorname{sign}\left[R_{h}(u)\right] u$.

Let $x_{h}(u)$ be a simple regular point of $\mathcal{H}_{h}$. As the point $x_{h}(u)$ is regular, the curvature function of $\mathcal{H}_{h}$ is nonzero at $u: R_{h}(u) \neq 0$. Recall that $R_{h}(u)$ is the product of the principal radii of curvature $R_{h}^{1}(u), \ldots, R_{h}^{n}(u)$ of $\mathcal{H}_{h}$ at $u$, which are defined as the eigenvalues of $x_{h}$ at $u$. Denote by $p$ (resp. $q$ ) the number of principal radii of curvature of $\mathcal{H}_{h}$ at $u$ that are positive (resp. negative), $\left((p, q) \in \mathbb{N}^{2}\right.$ and $\left.p+q=n\right)$.

Let us consider the variation of $\chi_{h}^{+}(x)$ when $x$, moving on the normal line to $\mathcal{H}_{h}$ at $x_{h}(u)$, crosses $\mathcal{H}_{h}$ at $x_{h}(u)$ in the direction of transverse orientation (that is, in the direction of $(-1)^{q} u$ ). We first consider the case where the sectional curvature $\sigma_{x_{h}(u)}$ of $\mathcal{H}_{h}$ at $x_{h}(u)$ is positive (i.e., $(p, q)=(n, 0)$ or $(0, n)$ ). In the sequel of the proof, $B^{n}$ will denote an open $n$-ball. If $q=0$, then the effect of the crossing on $\chi_{h}^{+}(x)$ is to add $\chi\left(B^{n}\right)-\chi\left(\mathbb{S}^{n}\right)$, that is -1 , to $\chi_{h}^{+}(x)$. If $q=n$, then the effect of the crossing on $\chi_{h}^{+}(x)$ is to add $(-1)^{n+1} \chi\left(B^{n}\right)$, that is -1 , to $\chi_{h}^{+}(x)$. Thus, in both cases, the effect of this crossing in the direction of transverse orientation is that $\chi_{h}^{+}(x)$ decreases by one unit.

We now turn to the case where $p$ and $q$ are nonzero. If we consider $\left(h_{x}\right)^{-1}(\{0\})$, which is a (not necessarily connected) smooth orientable hypersurface of $\mathbb{S}^{n}$ for any $x \in \mathbb{R}^{n+1}-\mathcal{H}_{h}\left(\right.$ since $\nabla h_{x}(u) \neq 0$ whenever $h_{x}(u)=0$ ), the effect of 
the crossing in the direction of transverse orientation can then be viewed as a surgery performed on the hypersurface. If $q$ is even (resp. odd), the "surgery" consists in cutting out a piece of hypersurface homeomorphic to $\mathbb{S}^{q-1} \times D^{p}$ (resp. $D^{q} \times \mathbb{S}^{p-1}$ ) and replacing it by a piece of hypersurface homeomorphic to $D^{q} \times \mathbb{S}^{p-1}$ (resp. $\mathbb{S}^{q-1} \times D^{p}$ ), where $D^{m+1}$ is the closed $m$-ball bounded by $\mathbb{S}^{m},(m \in \mathbb{N})$. Recall that such a surgery is possible by the fact that $\mathbb{S}^{p-1} \times \mathbb{S}^{q-1}$ can be regarded as the boundary of $\mathbb{S}^{q-1} \times D^{p}$ or as the boundary of $D^{q} \times \mathbb{S}^{p-1}$. When we consider $\left(h_{x}\right)^{-1}([0,+\infty[)$, the effect of the "surgery" is to remove (resp. to add) a cell complex that is homeomorphic to $D^{p} \times B^{q}$ if $q$ is even (resp. odd). Since Euler characteristic is multiplicative under cross products, the effect of the crossing on $\chi_{h}^{+}(x)$ is thus to add $(-1)^{q+1} \chi\left(B^{q}\right)$, that is -1 .

Proof of Corollary 5. By Theorem 4 , if $n+1$ is even, for every $x \in \mathbb{R}^{n+1} \backslash \mathcal{H}_{h}$, $i_{h}(x)=1+\chi_{h}^{-}(x)=\chi_{h}^{+}(x)+1$ and hence $i_{h}(x)=1+\frac{1}{2}\left(\chi_{h}^{-}(x)+\chi_{h}^{+}(x)\right)$. Since $\chi_{h}^{-}(x)+\chi_{h}(x)+\chi_{h}^{+}(x)=\chi\left(\mathbb{S}^{n}\right)=1+(-1)^{n}$, it follows that $i_{h}(x)=$ $1-\frac{1}{2} \chi_{h}(x)$.

Now, if $n+1$ is odd then, for every $x \in \mathbb{R}^{n+1} \backslash \mathcal{H}_{h}, i_{h}(x)=1-\chi_{h}^{-}(x)=$ $\chi_{h}^{+}(x)-1$ and hence $i_{h}(x)=\frac{1}{2}\left(\chi_{h}^{+}(x)-\chi_{h}^{-}(x)\right)$.

Proof of Theorem 6. We shall give later a proof valid in any dimension $n+1$, $\left(n \in \mathbb{N}^{*}\right)$. But, in order to deal with the case of cusp points, we present a slightly different proof in the plane.

Let $K, L \subset \mathbb{R}^{2}$ be convex bodies of class $C_{+}^{\omega}$ such that $\mathcal{H}_{h}$ is representing the formal difference $K-L$ in $\mathbb{R}^{2}$. We shall denote by $k$ and $l$ their respective support functions. Following the proof of Proposition 8 for $n+1=2$, we get

$$
\mathbf{1}_{h}(x)=1-\chi[(K+\{-x\}) \cap \partial L],
$$

since $x:=x_{h}(u)=x_{k}(u)+\left(-x_{l}(u)\right) \in K+(-L)$.

Note that $\partial(K+\{-x\})$ and $\partial L$ are internally tangent at the point $x_{l}(u)$ since $x_{l}(u)=x_{k_{x}}(u)$, where $k_{x}(u):=k(u)-\langle x, u\rangle,\left(u \in \mathbb{S}^{1}\right)$. Here 'internally' means that the two convex curves lie in the same side of their common tangent. Since $x:=x_{h}(u)$ is assumed to be a regular point of $\mathcal{H}_{h}$, we have $R_{h}(u) \neq 0$ and thus $R_{k_{x}}(u) \neq R_{l}(u)$.

If $R_{h}(u)>0$, then $R_{k_{x}}(u)>R_{l}(u)$, so that, in a neighborhood of the tangent point, $(\partial L) \backslash\left\{x_{l}(u)\right\}$ lie in the interior of $K+\{-x\}$. It follows that

$$
\chi[(K+\{-x\}) \cap \partial L]=\frac{1}{2}(\chi[\partial(K+\{-x\}) \cap \partial L]-1)=\frac{1}{2} n_{h}^{\prime}(x),
$$

where $n_{h}^{\prime}(x)=\chi\left(\left\{v \in \mathbb{S}^{1}-\{u\} \mid h_{x}(v)=0\right\}\right)$. Thus $\mathbf{1}_{h}(x)$ is then equal to $1-\frac{1}{2} n_{h}^{\prime}(x)$, which is the value taken by $i_{h}$ on the connected component of $\mathbb{R}^{2} \backslash \mathcal{H}_{h}$ towards which the unit normal vector $-u$ is pointing to.

If $R_{h}(u)<0$, then $R_{k_{x}}(u)<R_{l}(u)$, so that, in a neighborhood of the tangent point, $(\partial(K+\{-x\})) \backslash\left\{x_{l}(u)\right\}$ lie in the interior of $L$. It follows that 


$$
\chi[(K+\{-x\}) \cap \partial L]=\frac{1}{2}(\chi[\partial(K+\{-x\}) \cap \partial L]+1)=\frac{1}{2} n_{h}^{\prime}(x)+1,
$$

where $n_{h}^{\prime}(x)=\chi\left(\left\{v \in \mathbb{S}^{1}-\{u\} \mid h_{x}(v)=0\right\}\right)$. Thus $\mathbf{1}_{h}(x)$ is then equal to $-\frac{1}{2} n_{h}^{\prime}(x)$, which is the value taken by $i_{h}$ on the connected component of $\mathbb{R}^{2} \backslash \mathcal{H}_{h}$ towards which the unit normal vector $-u$ is pointing to.

Following the same approach for a simple cusp point $c:=x_{h}(v)$ and noticing that $R_{h}=R_{k_{x}}-R_{l}$ changes sign at $v$, we obtain

$$
\mathbf{1}_{h}(c)=1-\frac{1}{2} n_{h}^{\prime}(c),
$$

where $n_{h}^{\prime}(c)=\chi\left(\left\{v \in \mathbb{S}^{1}-\{v\} \mid h_{x}(v)=0\right\}\right)$, which is the required value for $\mathbf{1}_{h}(c)$.

Proof of Theorem 7. Let $K, L \subset \mathbb{R}^{n+1}$ be convex bodies of class $C_{+}^{\omega}$ such that $\mathcal{H}_{h}$ is representing the formal difference $K-L$ in $\mathbb{R}^{n+1}$. Denote by $k$ and $l$ their respective support functions. Following the proof of Proposition 8 , we get

$$
\mathbf{1}_{h}(x)=(-1)^{n+1}(1-\chi[(K+\{-x\}) \cap \partial L]),
$$

since $x:=x_{h}(u)=x_{k}(u)+\left(-x_{l}(u)\right) \in K+(-L)$. Note that $\partial(K+\{-x\})$ and $\partial L$ are internally tangent at the point $x_{l}(u)$ since $x_{l}(u)=x_{k_{x}}(u)$, where $k_{x}(u):=k(u)-\langle x, u\rangle,\left(u \in \mathbb{S}^{n}\right)$.

The result is the consequence of the following four observations:

(i) The proof of Proposition 8 can be adapted to obtain $\chi[(K+\{-x\}) \cap \partial L]=$ $\chi_{h}(x)+\chi_{h}^{+}(x)$ in the present case;

(ii) $\chi_{h}^{-}(x)+\chi_{h}(x)+\chi_{h}^{+}(x)=\chi\left(\mathbb{S}^{n}\right)=1+(-1)^{n}$;

(i) At $x=x_{h}(u), \chi_{h}^{-}: \mathbb{R}^{n+1} \rightarrow \mathbb{Z}, p \mapsto \chi\left[\left(h_{p}\right)^{-1}(]-\infty, 0[)\right]$ takes the same value as the one it takes on the connected component of $\mathbb{R}^{n+1} \backslash \mathcal{H}_{h}$ towards which $-u$ is pointing to;

(ii) On this connected component, $i_{h}(p)=1+(-1)^{n+1} \chi_{h}^{-}(p)$ by Theorem 4 .

\section{Further remarks and applications}

\section{Euler characteristic of an analytic hedgehog}

Let $\mathcal{H}_{h} \subset \mathbb{R}^{n+1}$ be an analytic hedgehog. Define its Euler characteristic by:

$$
\chi\left(\mathcal{H}_{h}\right):=\int_{\mathbb{R}^{n+1}} \mathbf{1}_{h}(x) d \chi(x) .
$$

Proposition 13 Any analytic hedgehog of $\mathbb{R}^{n+1}$ has Euler characteristic 1. 
Proof of proposition. Let $\mathcal{H}_{h}$ be a $C^{\omega}$-hedgehog of $\mathbb{R}^{n+1}$ and let $K, L \subset \mathbb{R}^{n+1}$ be convex bodies of class $C_{+}^{\omega}$ such that $\mathcal{H}_{h}$ is representing the formal difference $K-L$. By the definitions of $\chi\left(\mathcal{H}_{h}\right)$ and $\mathbf{1}_{h}$, we have:

$$
\chi\left(\mathcal{H}_{h}\right):=\int_{\mathbb{R}^{n+1}}(-1)^{n+1}\left(\mathbf{1}_{K} * \mathbf{1}_{-\stackrel{o}{L}}\right)(x) d \chi(x) .
$$

Convolution behaves has expected in $C F\left(\mathbb{R}^{n+1}\right)$ [2] and by reversing the order of integration, we get immediately:

$$
\chi\left(\mathcal{H}_{h}\right)=(-1)^{n+1}\left(\int_{\mathbb{R}^{n+1}} \mathbf{1}_{K}(x) d \chi(x)\right)\left(\int_{\mathbb{R}^{n+1}} \mathbf{1}_{-L}^{o}(x) d \chi(x)\right),
$$

that is, $\chi\left(\mathcal{H}_{h}\right)=(-1)^{n+1} \chi(K) \chi(-\stackrel{o}{L})=(-1)^{n+1} \chi(D) \chi(\stackrel{o}{D})=1$, where $D$ is the closed $(n+1)$-ball bounded by $\mathbb{S}^{n}$ in $\mathbb{R}^{n+1},(n \in \mathbb{N})$.

Remark. For any analytic hedgehog $\mathcal{H}_{h} \subset \mathbb{R}^{n+1}, \chi\left(\mathcal{H}_{h}\right)$ can also be regarded as the Euler characteristic of the complementary of the unbounded connected component of $\mathbb{R}^{n+1}-\mathcal{H}_{h}$, which is a compact contractible tame set.

\section{Sturm-Hurwitz type theorems} form

The Sturm-Hurwitz theorem states that any continuous real function of the

$$
f(\theta)=\sum_{n=N}^{+\infty}\left(a_{n} \cos n \theta+b_{n} \sin n \theta\right)
$$

for some sequences of real numbers $\left(a_{n}\right)$ and $\left(b_{n}\right)$, has at least as many zeros as its first nonvanishing harmonics: $\#\{\theta \in[0,2 \pi[\mid f(\theta)=0\} \geq 2 N$.

For $C^{2}$-functions, this result is closely related to the index $i_{h}(x)$ of a $C^{2}$ hedgehog $\mathcal{H}_{h} \subset \mathbb{R}^{2}$ with respect to a point $x \in \mathbb{R}^{2} \backslash \mathcal{H}_{h}$ and to its relationship with the number of zeros of $h_{x}(u)=h(u)-\langle x, u\rangle,\left(u \in \mathbb{S}^{1}\right)$ [11, 16]. So, our results suggest that, in higher dimensions, Sturm-Hurwitz type theorems might resort to the Euler characteristic.

\section{Mixed volume of analytic hedgehogs}

As a consequence of Theorems 2 and 3, we have:

Given hedgehogs with support functions $h_{1}, \ldots, h_{n+1} \in C^{\omega}\left(\mathbb{S}^{n} ; \mathbb{R}\right)$, the real function $P: \mathbb{R}^{n+1} \longrightarrow \mathbb{R}$ given by

$$
P\left(\alpha_{1}, \ldots, \alpha_{n+1}\right):=v_{n+1}\left(\sum_{k=1}^{n+1} \alpha_{k} h_{k}\right)=\int_{\mathbb{R}^{n+1}}\left(\mathbf{1}_{\alpha_{1} h_{1}} * \ldots * \mathbf{1}_{\alpha_{n+1} h_{n+1}}\right)(x) d \lambda(x),
$$


where $\lambda$ denotes the Lebesgue measure on $\mathbb{R}^{n+1}$, is a homogeneous polynomial the coefficients of which are the mixed volumes of $\mathcal{H}_{h_{1}}, \ldots, \mathcal{H}_{h_{n+1}}$ up to a constant factors.

\section{On the mixed area of $K, L \subset \mathbb{R}^{2}$ when $L$ is centered}

Proposition 14 Let $K$ and $L$ be convex bodies of class $C_{+}^{2}$ in $\mathbb{R}^{2}$. Denote by $k$ and $l$ their respective support functions and let $k_{x}$ be the support function of $K$ with respect to $x: k_{x}(u):=k(u)-\langle x, u\rangle,\left(u \in \mathbb{S}^{1}\right)$. Denote by $-L$ the reflection of $L$ through the origin and $\widehat{l}$ its support function: $\widehat{l}(u)=l(-u),\left(u \in \mathbb{S}^{1}\right)$. We have:

$$
v_{2}(k, c(l))=\frac{1}{8} \int_{K+(-L)} n_{k-l}(x) d \lambda(x),
$$

where $c(l)$ is the centered part of $l$, that is, $c(l)=\frac{1}{2}(l+\hat{l})$ and $n_{k-l}(x)=$ $\#\left(k_{x}-l\right)^{-1}(\{0\})=\#\left\{u \in \mathbb{S}^{1} \mid k_{x}(u)=l(u)\right\}$. In particular, if $L$ is centered (i.e., centrally symmetric with respect to the origin), then

$$
v_{2}(K, L)=\frac{1}{8} \int_{K+L} n_{k-l}(x) d \lambda(x) .
$$

Proof. As we have recalled, for any $C^{2}$-hedgehog $\mathcal{H}_{h}$, we have [8]:

$$
\forall x \in \mathbb{R}^{2} \backslash \mathcal{H}_{h}, \quad i_{h}(x)=1-\frac{1}{2} n_{h}(x),
$$

where $n_{h}(x)$ denotes the number of cooriented support lines of $\mathcal{H}_{h}$ through $x$, that is, the number of zeros of $h_{x}: \mathbb{S}^{1} \rightarrow \mathbb{R}, u \mapsto h(u)-\langle x, u\rangle$. Therefore, we have:

$$
\forall x \in(K+(-L)) \backslash \mathcal{H}_{h}, \quad\left(i_{k+\widehat{l}}-i_{k-l}\right)(x)=\frac{1}{2} n_{k-l}(x) .
$$

By integrating over $K+(-L)$, we get:

$$
v_{2}(k+\hat{l})-v_{2}(k-l)=\frac{1}{2} \int_{K+(-L)} n_{k-l}(x) d \lambda(x) .
$$

Since $v_{2}(k+\hat{l})-v_{2}(k-l)=2 v_{2}(k, l+\widehat{l})-\left(v_{2}(l)-v_{2}(\hat{l})\right)$ and $v_{2}(\hat{l})=v_{2}(l)$, it follows that:

$$
v_{2}(k, c(l))=\frac{1}{8} \int_{K+(-L)} n_{k-l}(x) d \lambda(x) .
$$

Remark. This result gives a geometrical interpretation of the mixed area when one of the arguments is a centered convex body since $n_{k-l}(x)$ is the number of 
common support lines of $K+\{-x\}$ and $L$.

\section{In higher even dimensions}

Starting from Corollary 5, we can easily obtain the following result in much the same way.

Proposition 15 Let $K$ and $L$ be convex bodies of class $C_{+}^{\omega}$ in $\mathbb{R}^{n+1}$, where $n+1$ is even. Denote by $k$ and $l$ their respective support functions and let $k_{x}$ be the support function of $K$ with respect to $x: k_{x}(u):=k(u)-\langle x, u\rangle,\left(u \in \mathbb{S}^{n}\right)$. Denote by $-L$ is the reflection of $L$ through the origin and $\widehat{l}$ its support function: $\widehat{l}(u)=l(-u),\left(u \in \mathbb{S}^{n}\right)$. We have:

$$
v_{n+1}(k+\hat{l})-v_{n+1}(k-l)=\frac{1}{2} \int_{K+(-L)} \chi_{k-l}(x) d \lambda(x)
$$

where $\chi_{k-l}(x)=\chi\left[\left(k_{x}-l\right)^{-1}(\{0\})\right]=\chi\left[\left\{u \in \mathbb{S}^{n} \mid k_{x}(u)=l(u)\right\}\right]$.

\section{References}

[1] A.D. Alexandrov, On uniqueness theorem for closed surfaces (Russian), Doklady Akad. Nauk SSSR 22 (1939), 99-102.

[2] J. Curry, R. Ghrist and M. Robinson, Euler calculus with applications to signals and sensing. Advances in applied and computational topology. AMS short course on computational topology, New Orleans, 2011. Proceedings of Symposia in Applied Mathematics 70 (2012), 75-145.

[3] H. Geppert, Über den Brunn-Minkowskischen Satz. Math. Z. 42 (1937), $238-254$.

[4] H. Groemer, Minkowski addition and mixed volumes. Geom. Dedicata 6 (1977), 141-163.

[5] R. Langevin, G. Levitt and H. Rosenberg, Hérissons et multihérissons (enveloppes paramétrées par leur application de Gauss). Singularities, Banach Center Publ. 20 (1988), 245-253.

[6] C. MacLaurin and G. Robertson, Euler characteristic in odd dimensions. Aust. Math. Soc. Gaz. 30 (2003), 195-199.

[7] Y. Martinez-Maure, De nouvelles inégalités géométriques pour les hérissons. Arch. Math. 72 (1999), 444-453.

[8] Y. Martinez-Maure, Indice d'un hérisson : étude et applications. Publ. Mat. 44 (2000), 237-255.

[9] Y. Martinez-Maure, Contre-exemple à une caractérisation conjecturée de la sphère. C. R. Acad. Sci. Paris, Sér. I, 332 (2001), 41-44. 
[10] Y. Martinez-Maure, Hedgehogs and zonoids. Adv. Math. 158 (2001), 1-17.

[11] Y. Martinez-Maure, Les multihérissons et le théorème de Sturm-Hurwitz. Arch. Math. 80 (2003), 79-86.

[12] Y. Martinez-Maure, Théorie des hérissons et polytopes. C. R. Acad. Sci. Paris, Sér. I, 336 (2003), 241-244.

[13] Y. Martinez-Maure, A Brunn-Minkowski theory for minimal surfaces. Ill. J. Math. 48, 2004, 589-607.

[14] Y. Martinez-Maure, Geometric study of Minkowski differences of plane convex bodies. Canad. J. Math. 58 (2006), 600-624.

[15] Y. Martinez-Maure, A Sturm-type comparison theorem by a geometric study of plane multihedgehogs. Ill. J. Math. 52, 2008, 981-993.

[16] Y. Martinez-Maure, New notion of index for hedgehogs of $\mathbb{R}^{3}$ and applications. Eur. J. Comb. 31 (2010), 1037-1049.

[17] Y. Martinez-Maure, Uniqueness results for the Minkowski problem extended to hedgehogs. Cent. Eur. J. Math. 10, 2012, 440-450.

[18] P. McMullen, The polytope algebra. Adv. Math. 78 (1989), 76-130.

[19] G. Panina, Planar pseudo-triangulations, spherical pseudo-tilings and hyperbolic virtual polytopes. Technical Report math.MG:0607171, arXiv, 2006.

[20] A.V. Pukhlikov and A.G. Khovanskii, Finitely additive measures of virtual polytopes. St. Petersbg. Math. J. 4 (1993),337-356.

[21] H. Rosenberg and E. Toubiana, Complete minimal surfaces and minimal herissons. J. Differential Geom. 28 (1988), 115-132.

[22] R. Schneider, Convex Bodies: The Brunn-Minkowski Theory. Cambridge University Press, Cambridge (1993).

[23] P. Schapira, Operations on constructible functions. J. Pure Appl. Algebra 72 (1991), 83-93.

[24] L. van den Dries, Tame topology and o-minimal structures. London Math. Soc. Lecture NoteSeries, 248. Cambridge University Press, Cambridge (1998).

[25] 0. Viro, Some integral calculus based on Euler characteristic. Topology and geometry, Rohlin Semin. 1984-1986, Lect. Notes Math. 1346 (1988) $127-138$.

Institut Mathématique de Jussieu

UMR $7586 \mathrm{du}$ CNRS

Universités Paris 4 et Paris 7 
175 rue du Chevaleret

Paris, 75013, France

martinez@math.jussieu.fr 